However, some researchers would like to see the current FDA regulations beefed up. "Think of the attention that we place on the development of stem cells and all of the validation of cellular therapeutics," Gladwin says. "But because red cells are grandfathered in, we assume that every red cell is the same as another red cell."

\section{Getting personal}

Gladwin notes that there are more than 1,000 known mutations in red-cell enzymes, membrane proteins and hemoglobins, and he is investigating how various mutations could affect the storage quality of blood. $\mathrm{He}$ and his colleagues plan to analyze the DNA of blood samples taken from 14,000 volunteers to find variations in the genome that affect various blood attributes.

The number of days blood has been sitting on a shelf may be too blunt a measure of what the quality of a unit actually is, says
Zimring, because there's also so much variability among donors. One person's red cells may be pristine at 42 days, while another's could be effectively unusable at seven days, Zimring asserts. Researchers are studying what contributes to those differences. In one study published this year, researchers collected red blood cells from 13 men with the same blood type and tested the cells from 5 to 42 days. The team put mechanical stresses on the cells to see how easily they burst and found that the blood from one man with a metabolic disorder called hypertriglyceridemia showed dramatically escalating fragility over time ${ }^{3}$.

As some scientists tease apart the individual differences between blood donors, others continue to examine the effect of red blood cell transfusions. Koch has had a long interest in understanding how postoperative complications relate to the transfusion of red blood cells, including lung damage, heart attack and infections and reduced long-term survival ${ }^{4-6}$. She and her team wondered whether the length of storage of the red cell units could somehow contribute to the complications they had already observed, which led up to their 2008 retrospective study. Today, Koch is leading a randomized clinical trial involving 3,200 individuals undergoing procedures including heart bypass and valve repair and replacement, comparing those receiving blood stored less than 14 days to those receiving blood stored more than 20 days.

Another new trial is being co-led by Philip Spinella, a pediatric critical care specialist at St. Louis Children's Hospital in Missouri, and Marisa Tucci, of Sainte-Justine Hospital in Montreal. The trial, called ABC PICU, is just underway and will include more than 1,500 critically ill children ranging in age from three days to 16 years to figure out whether fresher red blood cells can reduce

\title{
What's in the Bag?
}

The preservatives within the bags, or 'units', of red blood cells have made a tremendous difference when it comes to extending shelf life-and new research suggests that one new mixture called SOLX could perhaps expand this to as long as 56 days.

Red cells in plastic bags, called blood units, are stored on refrigerated shelves until they're needed for transfusion or until they've reached their approved shelf life (42 days in the US and 35 days in many European countries). Additives such as sodium citrate, which binds calcium, keep red cells from clumping together. The sugar dextrose gives the red cells energy. Phosphate, meanwhile, preserves levels of ATP, the red cell's energy molecule. Adenine and mannitol help stop red cells from rupturing.

According to Haemonetics, the Massachusetts-based blood management company that's currently developing the preservative, SOLX (also known as AS-7) buffers $\mathrm{pH}$ levels, thus maintaining red cell metabolism. Two studies of SOLX are slated to be published online in the journal Transfusion this fall. One trial demonstrated that the percentage of transfused red cells recovered at 24 hours after the red cells were stored at 42 and 56 days and then transfused into healthy volunteers met FDA criteria. Red cells stored for 42 days in SOLX had less hemolysis and higher levels of ATP than blood stored in a standard storage solution.

The second study tested whether SOLX could extend the hold time of whole blood to 24 hours at room temperature before processing. (In the US, red cells have to be stored in the refrigerator within eight hours of collection.) The cell recovery after transfusion at 42 days showed low amounts of hemolysis and met FDA requirements. Although the recovery and hemolysis rates did not meet FDA requirements at 56 days for the cells that were held 18-24 hours before processing, a lower percentage of red cells stored in SOLX during that hold period ruptured compared with those that had been held in a standard solution.

The current US hold time of eight hours creates logistical problems for some blood centers because donated blood may often be collected hundreds of miles away from the handling labs. "People don't donate blood at midnight, so collection centers need second and third shifts to process the blood," Dumont says. Haemonetics is seeking approval from the FDA so that SOLX can be used to increase the hold time of collected blood to 24 hours. "A 24-hour hold could smooth out work flow," Dumont notes, "and reduce cost and waste in some places." 